\title{
Impulsividad y compulsividad en individuos dependientes de cocaína
}

\section{Impulsivity and compulsivity in cocaine dependent individuals}

\author{
María José Fernández-Serrano*; José Cesar Perales- \\ LÓPEZ ${ }^{\star *}$; LAURA MORENO-LÓPEZ ${ }^{* \star *}$; ANA SANTOS-RUIZ ${ }^{\star * *}$; \\ Miguel Pérez-García ${ }^{\star \star \star},{ }^{\star \star \star \star},{ }^{* \star \star \star *}$, Antonio Verdejo-

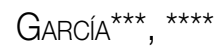

\author{
Departamento de Psicología. Universidad de Jaén. España. \\ ** Departamento de Psicología Experimental y Fisiología del Comportamiento. \\ Universidad de Granada. España.

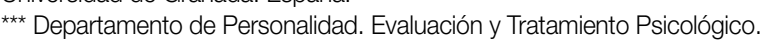 \\ Universidad de Granada. España.

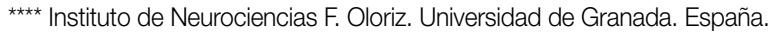 \\ ${ }^{\star * \star * \star}$ CIBERSAM. Universidad de Granada. España. \\ Enviar correspondencia a: \\ María José Fernández-Serrano \\ Departamento de Psicología, Facultad de Humanidades y Ciencias \\ de la Educación, Universidad de Jaén \\ Campus Las Lagunillas s/n, 23071 Jaén, España \\ E-mail: mifernan@ujaen.es \\ Teléfono: +34-953-212-126
}

recibido: Octubre 2011 aceptado: Enero 2012

\section{Resumen}

Recientes modelos neurobiológicos conciben la adicción como una transición del control de la conducta a lo largo del eje que va de la impulsividad a la compulsividad. La naturaleza dinámica de esta transición hace difícil detectar la posición de los adictos en este continuo, pero una de las aproximaciones mejor validadas es la caracterización de su estado mediante indices neuropsicológicos de impulsividad (o desinhibición) y compulsividad (o perseverancia). En esta investigación se pretende estimar la posición relativa de cada uno de los individuos en un grupo de pacientes dependientes de cocaina (PDC) en el eje impulsividadcompulsividad -en función de su rendimiento en indices de inhibición y perseverancia - y asociar su estatus neuropsicológico (impulsivo vs. compulsivo) con medidas de impulsividad-rasgo y severidad de exposición a la sustancia. 42 PDC y 65 controles sanos fueron evaluados mediante el cuestionario UPPS-P (para evaluar impulsividad), las pruebas Stroop y Go/No Go (para evaluar inhibición de respuesta) y los test de aplicación de estrategias y aprendizaje de inversión (para evaluar perseverancia). El 45\% de los PDC fueron clasificados como compulsivos. Este subgrupo presentaba puntuaciones significativamente más altas que el subgrupo impulsivo en los rasgos de falta de premeditación y falta de perseverancia. Un porcentaje sustancial de PDC presenta caracteristicas neuropsicológicas compatibles con un patrón de compulsividad. No se corroboró la idea de que los pacientes clasificados como compulsivos mostraran mayores niveles de severidad de la exposición a la cocaina; sin embargo, sí tienden a emitir respuestas poco meditadas y a desistir de tareas dirigidas a objetivos a largo plazo, tal y como muestran las pruebas de autoiforme de personalidad impulsiva.

\section{Abstract}

A recent theoretical approach describes addiction as a dynamic behavioural change process on the impulsivity-compulsivity axis. However, on the basis of current evidence, it is still difficult to establish a selective association between the course of addiction and individual transition along this axis. The aim of this study is to categorize each of the individuals in a sample of cocaine-dependent patients (CDI) as mainly impulsive or mainly compulsive, on the basis of their performance in neuropsychological inhibition and perseveration tests, and to test the association between the assigned category and their scores in trait impulsivity, and severity of cocaine addiction (measured by means of self-report assessment tools). A total of $42 \mathrm{CDI}$ and 65 healthy control individuals $(\mathrm{HCl})$ were assessed using the UPPS-P Scale (to explore trait impulsivity), the Stroop and Go/No Go (to assess response inhibition), and Revised-Strategy Application and Probabilistic Reversal tests (to assess response perseveration). Forty-five per cent of the CDIs were classified as compulsive, and this subgroup scored significantly higher than the impulsive group on the UPPS-P dimensions of lack of perseverance and lack of premeditation. A substantial proportion of CDIs can be classified as compulsive. No differences between compulsive and impulsive CDIs were found with regard to severity of exposure to cocaine; however, patients classified as compulsive by means of neuropsychological tasks are less perseverative in the pursuit of long-term objectives and more prone to make under-meditated decisions, as shown by trait impulsivity assessment questionnaires.

Key words: addiction, cocaine, impulsivity, compulsivity, drug use severity. 
E Manual Diagnóstico y Estadístico de los Trastornos Mentales (DSM-IV) define la dependencia de sustancias como un trastorno crónico y recurrente, caracterizado por una pérdida de control sobre el consumo y un patrón compulsivo de búsqueda de la sustancia a expensas de otras actividades más adaptativas para el individuo y beneficiosas para su entorno (American Psychiatric Association, 1994). De acuerdo con esta visión clínica, la investigación neurobiológica y neuropsicológica concibe la adicción como una transición desadaptativa del control de la conducta desde la impulsividad (conducta motivada por la recompensa espera$\mathrm{da}$, que caracteriza los estadios iniciales y es un marcador de vulnerabilidad de la adicción) y la compulsividad (conducta repetitiva y desvinculada de sus resultados -que caracteriza los estadios avanzados o de dependencia) (Dalley, Everitt y Robbins, 2011; Everitt y Robbins, 2005; Koob y Volkow, 2010; Lubman, Yucel y Pantelis, 2004). Esta transición entre las dos dimensiones neuropsicológicas está mediada por neuroadaptaciones en dos sistemas cerebrales que intercambian su protagonismo a lo largo del ciclo adictivo: la conducta impulsiva responde al disparo de señales de predicción de reforzamiento que se proyectan desde el estriado ventral hacia la corteza prefrontal, mientras que la compulsiva obedece a proyecciones descendentes desde el estriado dorsal a los efectores motores (Everitt y Robbins, 2005). Los modelos animales apoyan estos supuestos mostrando que los individuos premórbidamente impulsivos son más vulnerables a la autoadministración de cocaína en sus primeros contactos con la sustancia (Dalley et al., 2007), y más proclives a transitar de manera rápida a la dependencia, definida como reiteración de las conductas de consumo incluso cuando éstas van seguidas de consecuencias negativas progresivamente más graves (Belin, Mar, Dalley, Robbins y Everitt, 2008). Por tanto, la vulnerabilidad impulsiva favorece la escalada de consumo y ésta a su vez la consolidación de hábitos que se repiten automáticamente en presencia de estados de estrés o señales de consumo (Koob y Volkow, 2010).

Los hallazgos neuropsicológicos en humanos son también congruentes con esta idea. El consumo recreativo de cocaína y otros psicoestimulantes en individuos vulnerables pero no dependientes, está asociado con elevaciones de los rasgos de personalidad impulsiva (Leland, Arce, Feinstein y Paulus, 2006; Verdejo-García et al, 2010), con déficits del control inhibitorio (Colzato, van den Wildenberg y Hommel, 2007; Verdejo-García et al, 2010), y con incrementos de la activación funcional del núcleo caudado durante una tarea de predicción de recompensas (Leland et al., 2006). Por tanto, los consumidores recreativos de psicoestimulantes presentan caracteristicas neuropsicológicas asociadas con la impulsividad. En cambio, los estudios neuropsicológicos en consumidores crónicos revelan déficits de flexibilidad y conductas seriales o de perseverancia desvinculadas del objetivo inicial de la tarea (Ersche, Roiser, Robbins y Sahakian, 2008; Verdejo-García, Rivas-Pérez, Vilar-López y Pérez-García, 2007a; Woicik et al., 2011); que además correlacionan con mayores niveles de exposición al consumo de cocaína (FernándezSerrano, Pérez-García, Schmidt Río-Valle y Verdejo-García, 2010a, Fernández-Serrano, Pérez-García, Perales y VerdejoGarcía, 2010b, Fernández-Serrano, Perales, Moreno-López, Pérez-García y Verdejo-García, 2011) y con peor pronóstico de tratamiento (Verdejo-García et al., en prensa). Por tanto, los consumidores crónicos de psicoestimulantes presentan características neuropsicológicas de perseverancia/desvinculación del resultado asociadas con la compulsividad (Dalley et al., 2011). Aunque la convergencia de estos hallazgos apoya la relevancia del continuo neuropsicológico impulsividad-compulsividad, la naturaleza dinámica de esta transición hace difícil evaluar la posición de los pacientes con dependencia de cocaína (PDC) a lo largo de este continuo, pese a que esta estimación puede tener importantes implicaciones clínicas (Ersche et al., 2011; Verdejo-García et al., 2011).

Recientemente Dalley et al. (2011) han propuesto que un método útil para caracterizar la adicción a psicoestimulantes consistiría en la investigación del impacto relativo de la impulsividad-rasgo frente al ejercido por la exposición a las drogas abusadas (por ejemplo, la cantidad y la duración de consumo de cocaína) en medidas neuropsicológicas de inhibición de respuesta (relacionadas con la impulsividad) vs. medidas de perseverancia de respuesta (relacionadas con la compulsividad). Teóricamente, la impulsividad rasgo tendría un mayor impacto en los deterioros de inhibición de respuesta, mientras que la severidad de consumo tendría un mayor impacto en la perseverancia de respuesta (ver FernándezSerrano et al., 2011). Sin embargo, también existe evidencia de que determinadas facetas de la conducta impulsiva están significativamente asociadas con indicadores de compulsividad. En estudios animales, el rendimiento cognitivo en tareas relacionadas con la inatención y falta de premeditación se asocian con una transición más rápida hacia conductas compulsivas (Belin et al., 2008). Por otro lado, estudios factoriales en humanos indican que ciertas dimensiones de impulsividad (especialmente las relacionadas con los sistemas motivacionales de evitación del castigo) se agrupan junto con indicadores de compulsividad (Ersche et al., 2011; Meda et al., 2009).

Basándonos en la propuesta de Dalley et al (2011), en este estudio proponemos aplicar una nueva aproximación a la estimación de la posición dentro del continuo neuropsicológico impulsividad-compulsividad. Esta aproximación está basada en el cálculo del balance entre el rendimiento neuropsicológico en índices de desinhibición (como indicadores neuropsicológicos de impulsividad) y de perseverancia (como indicadores neuropsicológicos de compulsividad). Es importante hacer explícito que ambos constructos (impulsividad y compulsividad) son abordados como dimensiones neuropsicológicas que pueden tener relevancia clínica en diversas psicopatologias (Robbins, Gillan, Smith, de Wit y Ersche, en prensa), pero que en este caso se aplican al estudio de la adicción (excluyendo sus posibles comorbilidades). La implementación de esta estrategia consta de dos pasos: (1) estimar el deterioro relativo de cada paciente en índices neuropsicológicos de impulsividad y compulsividad (a partir de la comparación con un grupo de control, dado que estas pruebas 
carecen de un referente normativo; ver Fernández-Serrano et al., 2010b), y (ii) utilizar el balance de ejecución entre ambos tipos de medidas para clasificar a cada individuo en uno $u$ otro lado del continuo, en función de si el rendimiento se inclina relativamente hacia los déficits de inhibición (impulsivo) o hacia los déficits de perseverancia (compulsivo). En función de esta clasificación podemos comparar el impacto relativo de los rasgos de personalidad impulsiva -como medida de vulnerabilidad- y la severidad del consumo -como medida de exposición o toxicidad- entre estos dos subgrupos de pacientes (ver Dalley et al., 2011). En otras palabras, analizaremos la asociación entre la posición del paciente en el eje (que se definiría categóricamente como impulsivo o compulsivo) y medidas de impulsividad rasgo y de severidad de consumo de la droga (que se definirian dimensionalmente). Por tanto, las hipótesis que planteamos son: (i) aquellos individuos clasificados como compulsivos presentarán mayores niveles de exposición (cantidad y/o duración) al consumo de cocaína con respecto a aquellos clasificados como impulsivos, y (ii) aquellos individuos clasificados como compulsivos presentarán puntuaciones más elevadas en las facetas de la conducta impulsiva relacionadas con la falta de premeditación y la evitación del castigo (urgencia negativa).

\section{Método}

\section{Participantes}

Participaron 42 pacientes dependientes de cocaína (PDC), con edades comprendidas entre los 19 y 44 años (Media=28.93, Desviación típica=6.39), y 65 controles sanos (CS), con edades entre 23 y 41 años $(M=30.17, D T=4.98)$. Los PDC fueron reclutados durante su rehabilitación en la comunidad terapéutica de "Proyecto Hombre Granada". Intencionadamente, todos los participantes del estudio son hombres, dada la baja prevalencia de mujeres que entraban en tratamiento en el momento de iniciar el estudio. Todos los PDC permanecieron abstinentes durante al menos 15 días antes de la evaluación neuropsicológica, aunque la media de duración de abstinencia en este grupo era de 34.28 semanas ( $D T=22.01$ ), por lo que es posible garantizar la ausencia de alteraciones neuropsicológicas debidas a los efectos agudos o residuales asociados al consumo de cualquier sustancia; confirmada por análisis semanales de orina. Fueron excluidos de la muestra aquellos individuos previamente diagnosticados con algún trastorno del Eje I y/o del Eje II del DSM-IV (distinto a los relacionados con el uso y dependencia de sustancias). Asimismo fueron excluidos del estudio aquellos individuos que presentaran historia de daño cerebral traumático, alteraciones neurológicas, infecciosas o sistémicas que afectaran al sistema nervioso central. Ninguno de los PDC se encontraba recibiendo fármacos sustitutivos durante el curso del estudio.

Los CS fueron reclutados a través de anuncios en las agencias de empleo público por lo que, al igual que los PDC, todos ellos estaban en situación de desempleo durante la realización del estudio. Los criterios de selección para este grupo fueron similares a los del grupo de PDC, incorporando como criterio de inclusión la ausencia de consumo de drogas - pasado o actual, con la excepción del consumo social de alcohol (menos de diez bebidas alcohólicas por semana) y del consumo de tabaco. La media de consumo de alcohol de los sujetos controles era 25.52 unidades/mes $(D T=30.82)$ y la media de duración del uso de bebidas alcohólicas era 10.63 años $(D T=5.57)$.

\section{Instrumentos}

Para el cálculo del balance entre desinhibición (o impulsividad) y perseverancia (o compulsividad), se emplearon los siguientes índices:

\section{Desinhibición (Impulsividad)}

\section{Test de Interferencia Palabra-Color Stroop (Delis-Kaplan Executive Functions System; Delis, Kaplan y Kramer, 2001)}

Esta tarea de papel y lápiz está formada por cuatro partes, cada una de las cuales consta de 50 elementos. En la parte 1 (nombrar colores) se presenta manchas de diferentes colores y se pide a los participantes que nombren el color de cada una de estas manchas rápidamente e intentando no equivocarse. En la parte 2 (lectura) se presenta las palabras "rojo", "azul" y "verde" impresas en tinta negra y se pide a los participantes que las lean en voz alta. En la parte 3 (inhibición) se introduce el efecto de interferencia: las palabras "rojo", "azul" y "verde" están impresas en colores incongruentes (p. ej., "rojo" en azul), y se pide a los participantes que nombren el color e ignoren la palabra, facilitando la ocurrencia de errores de inhibición. La parte 4 (cambio) consta de estímulos semejantes a los de la parte 3, pero los participantes deben alternar entre "leer" y "nombrar color". Para la evaluación de la impulsividad, la principal variable dependiente de esta tarea es el número de errores cometidos en la parte 3 (errores de inhibición).

\section{Tarea Go/no go}

Utilizamos una versión informatizada de esta tarea (Verdejo-García, Perales y Pérez-García, 2007b). La tarea consta de 60 ensayos. En los primeros 30 ensayos (antes del cambio de criterio), se pide a los participantes que pulsen cualquier tecla tan rápido como sea posible, cuando el estímulo "go" (una letra concreta) está presente, y que no emitan ninguna respuesta (no pulsar ninguna tecla) cuando el estímulo "no go" (otra letra diferente) aparece en pantalla. La asignación de los estímulos a las condiciones "go" y "no go" fue contrabalanceada entre los sujetos. En los siguientes 30 ensayos de la tarea (tras el cambio) se pide a los participantes que cambien la asignación de respuesta del estímulo "go" al 
estímulo "no go"; en otras palabras, se les pide que respondan al estímulo que anteriormente era el estímulo "no go" y que no respondan al estímulo que anteriormente era el "go". La proporción de ensayos go vs. no go en ambas fases (antes y después del cambio) es de $7 / 3$. El intervalo entre estímulos (IEE) es de $100 \mathrm{~ms}$, y cada estímulo se presenta durante $1000 \mathrm{~ms}$. Después de cada respuesta se proporciona feedback auditivo (dos sonidos distintivos) para indicar si la respuesta ha sido correcta o incorrecta. Si el participante no responde dentro de los 1000 ms, estos dos sonidos se utilizan como feedback positivo (en los casos en que el estímulo es "no go" y, por tanto, el sujeto acierta no emitiendo respuesta), o como feedback negativo (en los casos en que el estímulo es "go" y el sujeto se lo salta). Las respuestas se clasificaron en éxitos (respondía en presencia de un ensayo go), falsas alarmas (respondian en presencia de un ensayo no go), rechazos correctos (no respondian en presencia de ensayos no go) y pérdidas (no respondian en presencia de ensayos go). La principal variable dependiente como medida de impulsividad en nuestro estudio fue la tasa de falsas alarmas, calculada como el cociente entre el número de falsas alarmas y el número total de ensayos no go (falsas alarmas + rechazos correctos).

\section{Perseveración (compulsividad)}

\section{Tarea de aprendizaje de inversión probabilistico (Probabilistic Reversal Learning Task)}

Esta tarea computarizada está basada en la tarea de aprendizaje de inversión descrita en Swainson et al. 2000 (ver una descripción pictórica en Verdejo-Garcia et al, 2010). La tarea se compone de 160 ensayos, en los que se presentan simultáneamente dos estímulos que sólo difieren en el color (dos cuadrados dibujados con líneas de colores distintos) y se pide al sujeto que seleccione cuál de los dos es correcto. En cada fase de la tarea, un estímulo es considerado "correcto", de manera que su selección es reforzada en la mayoría de los casos, y el otro estímulo es "incorrecto", y su selección es penalizada en la mayoria de las ocasiones. En las fases 1 y 2 de la tarea (ensayos 1-80), la selección del estímulo correcto se reforzaba en 8 de cada 10 casos, y se penalizaba en 2 de cada 10; mientras que la selección del estímulo incorrecto se penalizaba en 8 de cada 10 casos y se reforzaba en 2 de cada 10. Esto significa que en $20 \%$ de los ensayos el ordenador proporciona feedback falso, esto es, la selección de un estímulo correcto va seguida de un falso feedback negativo, y la selección de un estímulo incorrecto va seguida de un falso feedback positivo (para simplificar, seguiremos etiquetando los dos colores como "correcto" e "incorrecto" ya que, a priori, el primero es el que con mayor probabilidad será reforzado, y el último el que será menos reforzado). El feedback positivo y negativo era presentado acústicamente (mediante dos sonidos distintos) y suponía ganar o perder cinco puntos en la tarea. El número total de puntos conseguidos era presentado continuamente en una de las esquinas de la pantalla. Las fases 3 y 4 (ensayos 81-160) eran idénticos a la fase 1 y 2 , respectivamente, pero la proporción de reforzamiento para las respuestas correctas era del $70 \%$ y para las incorrectas era del $30 \%$. Además, el color correspondiente a la selección correcta y el correspondiente a la selección incorrecta cambiaba después de 40 ensayos (después de cada fase), esto es, el estímulo que era correcto previamente se torna incorrecto y viceversa. La principal variable dependiente utilizada en nuestro estudio como medida de compulsividad/perseverancia fue el número total de errores de perseverancia, es decir, el número de respuestas consecutivas incorrectas tras el cambio de contingencia (calculado de acuerdo a la descripción ofrecida por Ersche et al., 2008 y Swainson et al., 2000).

Test de Aplicación de Estrategias (R-SAT, Levine, Dawson, Boutet, Schwartz y Stuss, 2000; versión española, Verdejo-García et al., 2007a)

Se trata de una prueba multitarea de papel y lápiz, compuesta por tres subtareas simples (trazar figuras, copiar frases y numerar objetos) repartidas en seis montones - dos por tarea, A y B, cada uno de los cuales contiene 120 elementos. Los participantes deben realizar elementos de cada uno de estos seis montones con el objetivo de acumular el máximo número de puntos al cabo de 10 minutos. Los elementos de tamaño grande puntúan 0 puntos, mientras que los pequeños puntúan 100 puntos cada uno -por tanto, la mayoría de participantes esquiva los elementos grandes. Sin embargo, tanto los elementos grandes como los pequeños son de dificultad variable: algunos de ellos son fáciles y rápidos de completar (p. ej. su realización conlleva unos pocos segundos, por lo que son definidos como "elementos breves"), mientras que otros son muy laboriosos y consumen mucho tiempo (p. ej. pueden conllevar más de un minuto, por lo que son definidos como "elementos largos"). Por tanto, la estrategia más eficaz (que debe descubrir el participante) es la de completar sólo los elementos breves y saltarse los elementos largos. No obstante, para empezar a saltarse los elementos largos -que aparecen en las páginas avanzadas de cada montón (después de las páginas iniciales en las que todos son breves) el participante debe revertir el patrón de ejecución al que se ha ido habituando durante las páginas iniciales -consistente en rellenar todos los elementos en serie. Es por eso que la prueba se considera un índice de perseverancia -incapacidad de revertir la estrategia auto iniciada cuando el cambio en las contingencias lo requiere. La principal variable dependiente que empleamos en este estudio como medida de compulsividad-perseverancia es el porcentaje de elementos breves completado por los participantes en relación con el número total de elementos completados.

Para la medición de variables relacionadas con la vulnerabilidad a la adicción (personalidad impulsiva) y grado de exposición o neurotoxicidad (severidad del consumo de cocaína) se utilizaron los siguientes instrumentos: 
Escala de Evaluación del Comportamiento Impulsivo UPPS-P (Whiteside y Lynam, 2001; adaptación española de VerdejoGarcía et al., 2010)

Esta escala está formada por 59 items que permiten la evaluación de cinco dimensiones de personalidad que pueden desencadenar conductas impulsivas: urgencia negativa, (falta de) perseverancia, (falta de) premeditación, búsqueda de sensaciones y urgencia positiva (Smith, Fischer, Cyders, Annus y Spillane, 2007). La primera dimensión, urgencia negativa, evalúa la tendencia del individuos a ceder ante impulsos fuertes, específicamente cuando estos van acompañados de emociones negativas como depresión, ansiedad o ira. La segunda dimensión, (falta de) perseverancia, evalúa la habilidad del individuo para persistir en la realización de trabajos o en el cumplimento de obligaciones a pesar del aburrimiento o fatiga que puedan implicar. La tercera dimensión, (falta de) premeditación, evalúa la habilidad del individuo para pensar en las consecuencias potenciales de su comportamiento antes de llevarlo a cabo. La cuarta dimensión, búsqueda de sensaciones, evalúa la preferencia del individuo por la estimulación o excitación. La última dimensión, urgencia positiva, evalúa la tendencia del individuo a ceder ante impulsos cuando estos van precedidos de fuertes emociones positivas. Cada item era valorado siguiendo un formato tipo Likert, con 4 alternativas de respuesta, desde 1 (rotundamente de acuerdo) hasta 4 (rotundamente en desacuerdo). Utilizamos la puntuación total de cada una de las cinco dimensiones en los correspondientes análisis estadisticos.

Inventario de Investigación de la Conducta Adictiva (IRAB; Verdejo-García, López-Torrecillas, Aguilar de Arcos y PérezGarcía, 2005)

Es una entrevista estructurada que permite explorar la cantidad y duración del consumo de distintas drogas. Permite la obtención de medidas de cantidad de consumo mensual de cada una de las sustancias usadas (cantidad por mes) y de duración del consumo de éstas (en años). La Tabla 1 muestra los resultados descriptivos de nuestra muestra en cada una de estas variables.

Tabla 1. Puntuaciones descriptivas de los patrones de cantidad y duración del uso de drogas en la muestra de pacientes dependientes de cocaína (PDC)

\begin{tabular}{llll}
\hline \multirow{2}{*}{ Sustancias } & Variables & PDC & \\
\cline { 3 - 4 } & & Media & D.T. \\
\hline Cannabis & Porros por mes & 100.64 & 101.70 \\
& Duración (años) & 5.33 & 4.32 \\
Cocaine & Gramos por mes & 18.96 & 29.18 \\
& Duración (años) & 4.13 & 2.91 \\
MDMA & Pastillas por mes & 10.19 & 10.26 \\
& Duración (años) & 2.81 & 2.39 \\
Alcohol & Unidades estándar de alcohol por mes & 87.48 & 85.27 \\
& Duración (años) & 8.52 & 9.68 \\
\hline
\end{tabular}

Nota. Sólo se muestran los datos sobre el consumo de drogas usados por $>15 \%$ de los PDC incluidos en la muestra.

\section{Procedimiento}

Los participantes fueron evaluados individualmente entre Noviembre de 2008 y Septiembre de 2009 en una única sesión con una duración aproximada de 3 horas (incluyendo descansos). Los instrumentos incluidos en este estudio formaban parte de un protocolo más extenso de evaluación en PDC dirigido al estudio del rendimiento neuropsicológico en áreas de atención, memoria, funciones ejecutivas y procesamiento de emociones. Todos los participantes fueron informados sobre los objetivos, beneficios y posibles inconvenientes asociados a la participación en el estudio, y todos ellos firmaron un consentimiento informado que certificaba su participación voluntaria en el mismo. Al finalizar la evaluación recibieron un informe neuropsicológico sobre su rendimiento en las pruebas. Los CS recibieron una compensación de 40 por su participación en el estudio.

\section{Análisis estadísticos}

En primer lugar, exploramos las variables dependientes para detectar posibles datos perdidos. Algunos datos se perdieron debido a problemas técnicos durante su recolección. Como resultado, el tamaño de la muestra de PDC es $n=37$ en la UPPS-P, y en el caso de CS es de $n=56$ para las tareas Go/ No-go y Reversal, y $n=63$ para el test de Stroop.

Las comparaciones de rendimiento neuropsicológico entre ambos grupos han sido publicadas previamente en Fernández-Serrano et al. (2011), por lo que a continuación describimos exclusivamente el nuevo conjunto de análisis realizado especificamente para este estudio.

Comparaciones preliminares entre las variables demográficas de los grupos (PDC vs CS) mostraron que ambos grupos estaban igualados estadisticamente para la variable edad pero los CS tenían significativamente mayor número de años de escolaridad $[(M=17, S D=4.25)$ vs. $(M=11.87, S D=3.40)]$. Los años de escolaridad estaban significativamente correlacionadas con las dimensiones de la UPPS falta de premeditación, $(r=-0.250, p=0.011)$, falta de perseverancia $(r=-0.288$, $p=0.003)$ y urgencia positiva $(r=-0.400, p<0.001)$ pero no correlacionaba con ninguna de las variables neuropsicológicas. A pesar de ello, elegimos aplicar una aproximación estadistica conservadora a fin de controlar cualquier posible efecto de esta variable sobre el rendimiento de los participantes. De este modo, las puntuaciones de los test fueron transformados en puntuaciones estandarizadas resultantes de: (i) regresionar los años de educación (significativamente diferentes entre PDC y CS) sobre las variables dependientes, y (ii) salvar los residuos estandarizados, que fueron utilizados como variables dependientes en todos los análisis subsiguientes.

Para estimar la posición de cada PDC en el continuo empírico de impulsividad-compulsividad, los residuos estandarizados de sus índices de ejecución fueron transformados en puntuaciones $Z$, a partir de la media y desviación típica de las puntuaciones obtenidas por el grupo control (CS) en 
estos mismos índices (Fernández-Serrano, 2010b). De estos cálculos se obtuvieron dos puntuaciones $Z$ de desinhibición (Z-Stroop y Z-Go/No-go) y dos puntuaciones $Z$ de perseverancia (Z-Reversal y Z-RSAT), todas ellas en una misma escala. Seguidamente, sumamos las puntuaciones $Z$ de los dos índices de desinhibición, obteniendo así una puntuación global estandarizada de impulsividad, y las puntuaciones $Z$ de los dos índices de perseverancia, obteniendo de forma similar una puntuación global estandarizada de compulsividad. A continuación, calculamos el balance entre la puntuación Z-impulsividad y la puntuación Z-compulsividad (las puntuaciones positivas indicarian predominio del patrón de conducta impulsivo, mientras que las negativas indicarian predominio del patrón de conducta compulsivo en cada individuo). Este balance nos permite clasificar los PDC en individuos impulsivos o compulsivos.

Finalmente, previa comprobación de la normalidad de las distribuciones, llevamos a cabo pruebas $t$ de Student para explorar las diferencias en medidas de personalidad impulsiva (UPPS-P) y de severidad de consumo de cocaína y alcohol (la principal sustancia de co-abuso) entre los dos subgrupos obtenidos empíricamente (PDC impulsivos vs. PDC compulsivos).

\section{Resultados}

El $54.7 \%$ de los PDC se sitúa en el polo de Impulsividad del continuo, esto es, obtenían puntuaciones $Z$ de balance positivas, mientras que el $45.3 \%$ restante se ubica en el polo de Compulsividad (obtenían puntuaciones $Z$ de balance negativas)

Cuando comparamos ambos grupos en medidas de vulnerabilidad (personalidad impulsiva) y toxicidad (severidad acumulada), los resultados mostraron que los PDC compulsivos presentaban puntuaciones significativamente elevadas en las dimensiones de la UPPS-P falta de perseverancia $(p=.016)$ (ver figura 1) y falta de premeditación $(p=.018)$ (ver figura 2). En cambio, contrariamente a nuestra hipótesis de partida, los resultados no mostraron diferencias significativas entre ambos subgrupos en sus patrones de severidad de consumo de cocaina y alcohol (ver Tabla 2 y figura 3 ).

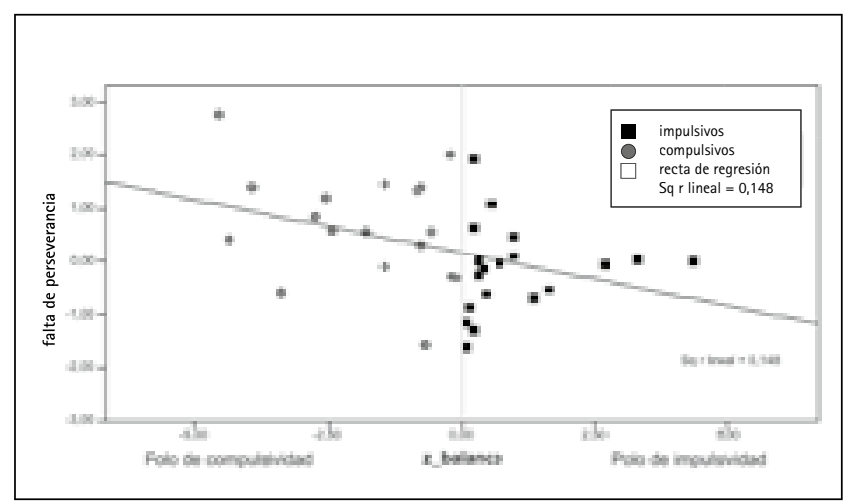

Figura 1. Falta de perseverancia en los PDC impulsivos vs. compulsivosInsertar Figura 1

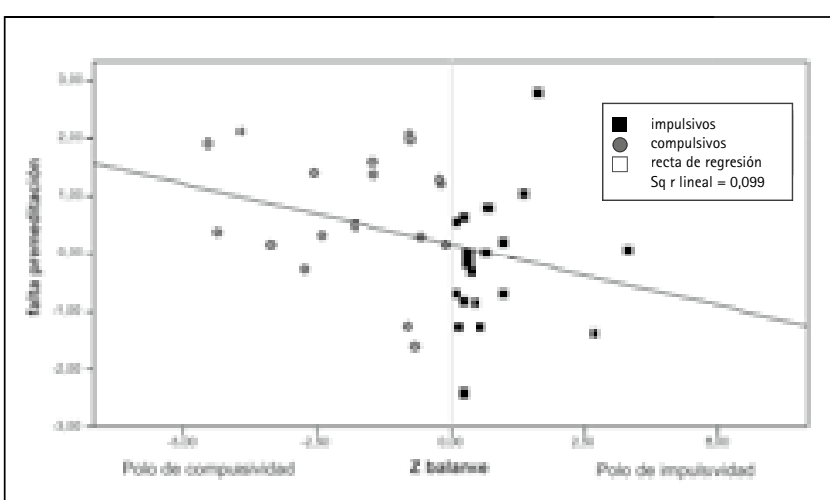

Figura 2. Falta de premeditación en los PDC impulsivos vs. compulsivos

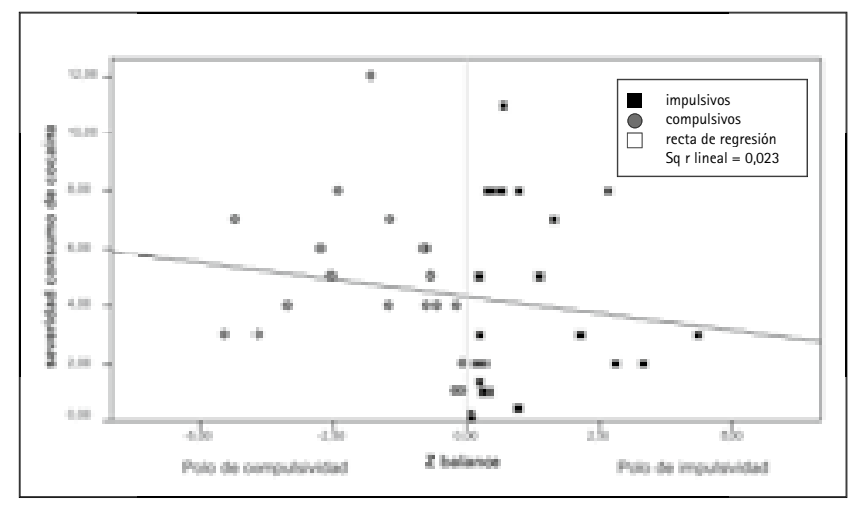

Figura 3. Severidad de consumo de cocaína en los PDC impulsivos vs. compulsivos

Tabla 2. Comparación entre los pacientes dependientes de cocaína (PDC) clasificados como impulsivos vs compulsivos en medidas de impulsividad-rasgo y patrones de consumo de droga

\begin{tabular}{llll}
\hline & Impulsivos $(\mathrm{n}=23)$ & Compulsivos $(\mathrm{n}=19)$ & $\mathrm{t}$ (valor $\mathrm{p})$ \\
\hline & Mean (SD) & Mean (SD) & \\
\hline Cantidad de cocaina & $21.63(36.03)$ & $11.73(13.26)$ & $-1.13(.264)$ \\
Duración de cocaina & $3.96(3.23)$ & $4.84(2.60)$ & $.958(.344)$ \\
Cantidad de alcohol & $174.17(161.63)$ & $155.42(175.20)$ & $-.360(.720)$ \\
Duración de alcohol & $9.52(5.99)$ & $9.63(5.58)$ & $.061(.952)$ \\
Urgencia negativa & $.30(1.13)$ & $.72(.70)$ & $1.31(.199)$ \\
Falta de premeditación & $-.22(1.19)$ & $.74(1.09)$ & $2.49(.018)$ \\
Falta de perseverancia & $-.17(.88)$ & $.65(1.02)$ & $2.54(.016)$ \\
Búsqueda de sensaciones & $.18(1.04)$ & $.32(1.12$ & $.370(.714)$ \\
Urgencia positiva & $.43(1.21)$ & $.78(.78)$ & $1.01(.317)$ \\
\hline
\end{tabular}

Nota. En negrita se muestran diferencias significativas entre grupos

\section{Discusión}

Nuestros resultados muestran que, tomando como referencia el rendimiento neuropsicológico de un amplio grupo control, los pacientes dependientes de la cocaína pueden clasificarse como impulsivos o compulsivos según su balance de deterioro en medidas de desinhibición vs. perseverancia. Un 
elevado porcentaje de los pacientes dependientes de cocaína en tratamiento se escoraron hacia el polo de compulsividad, caracterizado por conductas seriales y perseverativas desvinculadas de sus objetivos finalistas. Con respecto al subgrupo clasificado como "impulsivo", los consumidores "compulsivos" presentaban puntuaciones más altas en falta de premeditación y falta de perseverancia, indicativos de problemas de precipitación y tendencia a desistir de tareas dirigidas a objetivos a largo plazo. En cambio, contrariamente a nuestra hipótesis, los individuos "compulsivos" no se diferenciaron de los "impulsivos" en términos de severidad de consumo.

Mientras que la impulsividad es un fenómeno bien conocido y ampliamente abordado en la clínica de las adicciones (Economidou, Pelloux, Robbins, Dallew y Everitt, 2009; Moeller et al., 2001; Verdejo-García, Lawrence y Clark, 2008), las implicaciones clínicas asociadas al patrón de conducta compulsivo y las intervenciones terapéuticas dedicadas a su abordaje están notablemente menos desarrolladas. Por ejemplo, en un estudio reciente demostramos que los pacientes dependientes de opiáceos con altos niveles de compulsividad son menos sensibles a los efectos del craving sobre el control de la conducta (Verdejo-García et al., 2011); posiblemente porque sus conductas son más habituales y están mucho menos dirigidas por la previsión de la obtención de reforzadores (Hogarth, 2011). La detección de un alto porcentaje de consumidores compulsivos en una muestra representativa de los pacientes que inician tratamiento en Comunidad Terapéutica por consumo de cocaina -sin otras comorbilidades- puede ser de interés para el desarrollo de planes de intervención ajustados a las características neuropsicológicas de los pacientes drogodependientes.

La principal característica distintiva de la conducta compulsiva, de acuerdo con los resultados de este estudio, es la exacerbación de los rasgos de falta de premeditación y falta de perseverancia. La dimensión de falta de perseverancia refleja dificultades que se solapan en gran medida con los déficits típicamente observados en tareas de rendimiento continuo (Continuous Performance Tests o CPTs) y ha sido relacionada a nivel teórico con el Trastorno por Déficit de Atención e Hiperactividad (Whiteside y Lynam, 2001). Asimismo, esta dimensión correlaciona positivamente con la aparición de pensamientos no relacionados con la tarea en el curso de la ejecución otras tareas cognitivas (Gay, Rochat, Billieux, d'Acremont y van der Linden, 2008), un fenómeno teóricamente vinculado con el craving entendido como rumiación compulsiva (Hester y Garavan, 2005). Por otra parte, tanto la falta de perseverancia como la falta de premeditación correlacionan negativamente con el rendimiento en el Test de Emparejamiento de Figuras Familiares (Matching Familiar Figures Test; ver Perales, Verdejo-Garcia, Moya, Lozano y Pérez-García, 2009), que detecta la ocurrencia de respuestas prematuras - de un modo similar al modelo animal que permitió identificar a aquellos individuos impulsivos que posteriormente transitaron de un modo más rápido hacia la compulsividad y la dependencia (Belin et al., 2008; Dalley et al., 2007). Por tanto, la convergencia de los resul- tados animales y humanos nos permite proponer, de modo aún especulativo, que la falta de premeditación y la falta de perseverancia son dimensiones de personalidad relevantes, que confieren un mayor riesgo de progresión hacia patrones de perseverancia en PDC (ya que el grado de exposición a la sustancia no parece ser, en nuestro caso, un mediador importante en su aparición). Tampoco se detectaron las diferencias esperadas en el rasgo de urgencia negativa, que recoge la tendencia a cometer actos impulsivos bajo estados de malestar o estrés y que se ha asociado con indicadores de severidad de consumo (Verdejo-García et al., 2007b). Es posible que esta faceta de la conducta impulsiva esté más vinculada a dimensiones neuropsicológicas dependientes del feedback afectivo, como es el caso de la toma de decisiones (Verdejo-García y Bechara, 2009).

Nuestro estudio tiene una serie de fortalezas, entre las que destacan el reclutamiento de una muestra clínicamente bien caracterizada de PDC en situación de abstinencia controlada y el empleo de instrumentos neuropsicológicos de alta fiabilidad, precisión y especificidad en la detección de déficits de inhibición y perseverancia. Respecto a las limitaciones de este estudio podemos destacar las diferencias en años de escolaridad entre los PDC y los individuos no consumidores. En relación a esta limitación algunos expertos han planteado que estas discrepancias son inherentes a la población con dependencia de tal forma que su eliminación también podría restar relevantes características relacionadas con la adicción (Adams, Brown y Grant, 1985). A pesar de ello aplicamos un enfoque estadístico conservador a fin de controlar dichas diferencias. Por otra parte, una limitación relevante en este estudio es la elección de una aproximación categorial al balance entre los polos de impulsividad y compulsividad. Futuros estudios deberían comparar esta aproximación con un paradigma de carácter más dimensional, 0 bien replicar nuestras propuestas en estudios longitudinales que sigan la transición entre impulsividad y compulsividad.

\section{Reconocimientos}

La investigación descrita en este trabajo ha sido financiado por un proyecto del Ministerio de Educación a través del Plan Nacional FPU (referencia AP 2005-1411) para la primera autora, un proyecto del Ministerio de Ciencia e Innovación (MICINN), a través de la Dirección General de Investigación y Gestión del Plan Nacional de I+D+i (referencia PSI2009-13133) para el segundo autor, por el proyecto P07.HUM 03089 a través de la Junta de Andalucía (referencia SEJ 2006-8278) y por el proyecto COPERNICO a través del Plan Nacional sobre Drogas (2009) para el último autor.

\section{Conflicto de intereses}

Los autores no tienen conflictos de intereses que declarar. 


\section{Referencias}

Adams, K. M., Brown, G. G. y Grant, I. (1985). Analysis of covariance as a remedy for demographic mismatch of research subject groups, some sobering simulations. Journal of Clinical and Experimental Neuropsychology, 7, 445-462. doi: 10.1080/01688638508401276

American Psychiatric Association (1994). Diagnostic and Statistical Manual of Mental Disorders ( $4^{\text {th }}$ ed.). Washington, DC. doi: 10.1176/appi.books.9780890423349

Belin, D., Mar, A. C., Dalley, J. W., Robbins, T. W. y Everitt, B. J. (2008). High impulsivity predicts the switch to compulsive cocainetaking. Science, 320, 1352-1355. doi: 10.1126/science. 1158136

Colzato, L. S., van den Wildenberg, W. P. M. y Hommel, B. (2007). Impaired inhibitory control in recreational cocaine users. PLOS ONE, 2, 1143. doi: 10.1371/journal.pone.0001143

Dalley, J. W., Everitt, B. J. y Robbins, T. W. (2011). Impulsivity, compulsivity and top-down cognitive control. Neuron, 69, 680694. doi: 10.1016/j.neuron.2011.01.020

Dalley, J. W., Fryer, T. D., Brichard, L., Robinson, E. S., Theobald, D. E., Lääne, K., Peña, Y., Murphy, E., Shah, Y., Probst, K., Abakumova, I., Aigbirhio, F. I., Richards, H. K., Hong, Y., Baron, J. C., Everitt, B. J., Robbins, T. W. (2007). Nucleus accumbens D2/3 receptors predict trait impulsivity and cocaine reinforcement. Science, 315, 12671270. doi: 10.1126/science. 1137073

Delis, D. C., Kaplan, E., Kramer, J. H. (2001). Delis-Kaplan executive function system. San Antonio, Texas: Psychological Corporation.

Economidou, D., Pelloux, Y., Robbins, T. W., Dalley, J. W. y Everitt, B. J. (2009). High impulsivity predicts relapse to cocaine-seeking after punishment-induced abstinence. Biological Psychiatry, 65, 851856. doi: 10.1016/j.biopsych.2008.12.008

Ersche, K. D., Barnes, A., Simon Jones, P., Morein-Zamir, S., Robbins, T. W. y Bullmore, E. T. (2011). Abnormal structure of frontostriatal brain systems is associated with aspects of impulsivity and compulsivity in cocaine dependence. Brain, 134, 2013-2024. doi: 10.1093/brain/awr138

Ersche, K. D., Roiser, J. P., Robbins, T. W. y Sahakian, J. (2008). Chronic cocaine but not chronic amphetamine use is associated with perseverative responding in humans. Psychopharmacology, 197, 421-431. doi: 10.1007/s00213-007-1051-1

Everitt, B. J., y Robbins, T. W. (2005). Neural systems of reinforcement for drug addiction, from action to habits to compulsion. Nature Neuroscience, 8, 1481-1489. doi: 10.1038/nn1579

Fernández-Serrano, M. J., Perales, J. C., Moreno-López, L., Pérez-Garcia, M. y Verdejo-Garcia, A. (2011). Neuropsychological profiling of impulsivity and compulsivity in cocaine dependent individuals. Psychopharmacology, 219, 673-683. doi: 10.1007/s00213-0112485-z

Fernández-Serrano, M. J., Pérez-Garcia, M., Perales, J. C. y VerdejoGarcia, A. (2010b). Prevalence of executive dysfunction in cocaine, heroin and alcohol users enrolled in therapeutic communities. European Journal of Pharmacology, 626, 104-112. doi: 10-1016/j. ejphar.2009.10.019

Fernández-Serrano, M. J., Pérez-Garcia, M., Schmidt Río-Valle, J. y Verdejo-García, A. (2010a). Neuropsychological consequences of alcohol and drug abuse on different components of executive functions. Journal of Psychopharmacology, 24, 1317-1332. doi: 10.1177/0269881109349841

Gay, P., Rochat, L., Billieux, J., d'Acremont, M. y Van der Linden, M. (2008). Heterogeneous inhibition processes involved in different facets of self-reported impulsivity, evidence from a community sample. Acta Psychologica, 129, 332-339. doi: 10.1016/j. actpsy.2008.08.010

Hester, R. y Garavan, H. (2005). Working memory and executive function, the influence of content and load on the control of attention. Memory \& Cognition, 33, 221-233. doi: 10.3758/ BF03195311

Hogarth, L. (2011). The role of impulsivity in the aetiology of drug dependence, reward sensitivity versus automaticity. Psychopharmacology, 2015, 567-580. doi: 10.1007/s00213-011-2172-0

Koob, G. F. y Volkow, N. D. (2010). Neurocircuitry of addiction. Neuropsychopharmacology, 35, 217-238. doi: 10.1038/ npp.2009.110

Leland, D. S., Arce, E., Feinstein, J. S. y Paulus, M. P. (2006). Young adult stimulant users' increased striatal activation during uncertainty is related to impulsivity. Neuroimage, 33, 725-731. doi: 10.1016/j. neroimage.2006.07.011

Levine, B., Dawson, D., Boutet, I., Schwartz, M. L, y Stuss, D. T. (2000). Assessment of strategic self-regulation in traumatic brain injury, Its relationship to injury severity and psychosocial outcome. Neuropsychology, 14, 491-500. doi: 10.1037/0894-4105.14.4.491

Lubman, D. I., Yucel, M. y Pantelis, C. (2004). Addiction, a condition of compulsive behaviour? Neuroimaging and neuropsychological evidence of inhibitory dysregulation. Addiction, 99, 1491-1502. doi: 10.1111/j.1360-0443.2004.00808.x

Meda, S. A., Stevens, M. C., Potenza, M. N., Pittman, B., Gueorquieva, R., Andrews, M. M., Thomas, A. D., Muska, C., Hylton, J. L. y Pearlson, G. D. (2009). Investigating the behavioural and self-report constructs of impulsivity domains using principal component analysis. Behavioral Pharmacology, 20, 390-399. doi: 10.1097/ FBP.0b013e32833113a3

Moeller, F. G., Dougherty, D. M., Barratt, E. S., Schmitz, J. M., Swann, A. C. y Grabowski, J. (2001). The impact of impulsivity on cocaine use and retention in treatment. Journal of Substance Abuse Treatment, 21, 193-198. doi: 10.1016/S0740-5472(01)00202-1

Perales, J. C., Verdejo-García, A., Moya, M., Lozano, 0. y Pérez-García, M. (2009). Bright and dark sides of impulsivity, performance of women with high and low trait impulsivity on neuropsychological tasks. Journal of Clinical and Experimental Neuropsychology, 31, 927-944. doi: 10.1080/13803390902758793

Robbins, T. W., Gillan, C. M., Smith, D. G., de Wit, S. y Ersche, K. D. (en prensa). Neurocognitive endophenotypes of impulsivity and compulsivity, towards dimensional psychiatry. Trends of Cognitive Science. doi: 10.1016/j.tics.2011.11.009

Smith, G. T., Fischer, S., Cyders, M. A., Annus, A. M. y Spillane, N. S. (2007). On the validity and utility of discriminating among impulsivity-like traits. Assessment, 14, 155-170. doi: 10.1177/1073191106295527

Swainson, R., Rogers, R. D., Sahakian, B. J., Summers, B. A., Polkey, C. E. y Robbins, T. W. (2000). Probabilistic learning and reversal deficits in patients with Parkinson's disease or frontal or temporal lobe lesions, possible adverse effects of dopaminergic medication. Neuropsychologia, 38, 596-612. doi: 10.1016/S00283932(99)00103-7

Verdejo-Garcia, A. y Bechara, A. (2009). A somatic marker theory of addiction. Neuropharmacology, 56, 48-62. doi: 10.1016/j. neuropharm.2008.07.035

Verdejo-Garcia, A., Lawrence, A. J. y Clark, L. (2008). Impulsivity as a vulnerability marker for substance-use disorders: review of 
findings from high-risk research, problem gamblers and genetic association studies. Neuroscience and Biobehavioral Reviews, 32, 777-810. doi: 10.1016/j.neubiorev.2007.11.003

Verdejo-García, A., López-Torrecillas, F., Aguilar de Arcos, F. y PérezGarcia M. (2005). Differential effects of MDMA, cocaine, and cannabis use severity on distinctive components of the executive functions in polysubstance users, a multiple regression analysis. Addiction Behaviors, 30, 89-101. doi: 10.1016/j. addbeh.2004.04.015

Verdejo-García, A., Lubman, D., Schwerk, A., Vilar-López, R., Roffel, K., MacKenzie, T. y Yucel, M. (en prensa). Effect of craving induction on inhibitory control in opiate dependence. Psychopharmacology. doi: 10.1007/s00213-011-2512-0

Verdejo-García, A., Perales, J. C. y Pérez-Garcia, M. (2007b). Cognitive impulsivity in cocaine and heroin polysubstance abusers. Addiction Behaviors, 32, 950-966. doi: 10.1016/j. addbeh.2006.06.032

Verdejo-García, A., Rivas-Pérez, C., Vilar-López, R. y Pérez García, M. (2007a). Strategic self-regulation, decision-making and emotion processing in polysubstance abusers in their first year of abstinence. Drug and Alcohol Dependence, 86, 139-146. doi: 10.1016/j.drugalcdep.2006.05.024

Verdejo-Garcia, A., Sánchez-Fernández, M. M., Alonso-Maroto, L. M., Fernández-Calderón, F., Perales, J. C., Lozano, 0. y Pérez-Garcia, M. (2010). Impulsivity and executive functions in polysubstanceusing rave attenders. Psychopharmacology, 210, 377-392. doi: 10.1007/s00213-010-1833-8

Whiteside, S. P. y Lynam, D. R. (2001). The five factor model and impulsivity, using a structural model of personality to understand impulsivity. Personality and Individual Differences, 30, 669-689. doi: 10.1016/S0191-8869(00)00064-7

Woicik, P. A., Urban, C., Alia-Klein, N., Henry, A., Maloney, T., Telang, F., Wang, G. J., Volkow, N. D. y Goldstein, R. Z. (2011). A pattern of perseveration in cocaine addiction may reveal neurocognitive processes implicit in the Wisconsin Card Sorting Test. Neuropsychologia, 49, 1660-1669. doi: 10.1016/j. neuropsychologia.2011.02.037 
